\title{
Clinical Trial Phases
}

\author{
Vicki L. Mahan \\ Department of Pediatric Cardiothoracic Surgery, St. Christopher's Hospital for Children/Drexel University \\ College of Medicine, Philadelphia, USA \\ Email: Vicki.mahan@tenethealth.com
}

Received 15 November 2014; revised 14 December 2014; accepted 22 December 2014

Copyright (C) 2014 by author and Scientific Research Publishing Inc.

This work is licensed under the Creative Commons Attribution International License (CC BY).

http://creativecommons.org/licenses/by/4.0/

(c) (i) Open Access

\begin{abstract}
Developers of drugs, biologicals, and medical devices must ensure product safety, demonstrate medical benefit in people, and mass produce the product. Preclinical development starts before clinical trials and the main goals are to determine safety and effectiveness of the intervention. If preclinical studies show that the therapy is safe and effective, clinical trials are started. Clinical trial phases are steps in the research to determine if an intervention would be beneficial or detrimental to humans and include Phases 0 , I, II, III, IV, and V clinical studies. Understanding the basis of clinical trial phases will help researchers plan and implement clinical study protocols and, by doing so, improve the number of therapies coming to market for patients.
\end{abstract}

\section{Keywords}

\section{Clinical Phases, Clinical Phase Trials, Preclinical Trials, Federal Drug Administration}

\section{Introduction}

Developers of drugs, biologicals, and medical devices must ensure product safety, demonstrate medical benefit in people, and mass produce the product. Preclinical development starts before clinical trials and the main goals are to determine safety and effectiveness of the intervention. Research may include pharmacodynamics, pharmacokinetics, absorption, distribution, metabolism and excretion studies, and toxicity testing. During preclinical studies, in vitro and in vivo testing is performed. Toxicity includes studies of which organs are targeted and long-term carcinogenic effects or effects on mammalian reproduction. Two species of animals are normally used in drug development studies. Choice is determined on which animal gives the best correlation to human studies. Medical devices are usually studied in larger animal species. No Observed Adverse Effect Level (NOAEL), the level of exposure at which there is no biologically or statistically significant increase in the frequency or severity of any adverse effects in the exposed population when compared to its appropriate control, is established based on preclinical trials. These are used to determine initial Phase I clinical trial dosage levels on a mass active 
pharmaceutical ingredient (API) per mass patient basis.

If preclinical studies show that the therapy is safe and effective, clinical trials, defined as "scientifically controlled studies of the safety and effectiveness of a therapeutic agent using consenting human subjects", are started. The four possible outcomes are: 1) the new treatment has a large beneficial effect and is superior to standard treatment; 2) the new treatment is equivalent to standard treatment; 3) the new treatment is neither clearly superior nor clearly inferior to standard treatment; or 4) a new treatment is inferior to standard treatment. The US Food and Drug Administration (FDA) role begins after preclinical evaluation for safety and effectiveness. These prospective studies are designed to answer specific questions about biomedical or behavioral interventions and must adhere to the principles of good clinical practices (GCP) [1]-[5]. Classification of the trial may reflect how the researchers behave (observational versus interventional clinical trials), by their purpose (prevention, screening, diagnostic, treatment, quality of life, or expanded access clinical trials), or whether the trial design allows changes based on data accumulated during the trial (fixed versus adaptive clinical trials). Ten areas that are carefully assessed in these clinical studies are protection of human subjects, sampling, degree of masking, randomization, intention to treat analysis, selection of interventional and comparison groups, selection of end points, interpretation of results, trial duration, and selection of traditional versus equivalence testing. Randomized controlled trials (RCT) are the gold standard and are often used to evaluate the efficacy or effectiveness of various types of medical intervention and may provide information about adverse effects [6]-[10]. Classifications of RCT's include study design (parallel-group, crossover, cluster, or factorial), outcome of interest (efficacy versus effectiveness), and evaluation of a hypothesis (superiority, noninferiority, or equivalence) [11] [12]. The people being studied are randomly allocated to one of the different treatments that are under study. The ideal randomization process maximizes statistical power, minimizes selection bias, and minimizes allocation bias [13]-[17].

Clinical trial phases are steps in the research to determine if an intervention would be beneficial or detrimental to humans and include Phases 0, I, II, III, IV, and V clinical studies [18] [19] (Table 1). During Phase 0, pharmacodynamics and pharmacokinetics are determined. Safety studies are evaluated during Phase I, efficacy during Phase II, and confirmation of safety and efficacy during Phase III. Sentry studies are done in Phase IV and comparative effectiveness research and community-based research in Phase V. Although this sounds easy and straightforward, definitions and purposes of the different phases become muddied and studies to determine if a therapy should be used in the general population of patients may be complex and results difficult to interpret. Clinical trials may go so wrong that unplanned changes in the population studied, end points or analysis plan must be made [20]. Understanding the basis of clinical trial phases will help researchers plan and implement clinical study protocols and, by doing so, improve the number of therapies coming to market for patients.

\section{Phase 0 Clinical Trials}

In September 2003, the National Institutes of Health (NIH) announced a series of initiatives to address the growing crisis in moving new basic science discoveries to the market where they are available for patient use. One of the objectives was strengthening clinical research infrastructure [21]. This was followed by an FDA report issued in March 2004 analyzing the "Challenge and Opportunity on the Critical Path to New Medical Products" [22]. US Pharmaceutical R \& D Spending and the NIH Budget had increased dramatically between

Table 1. Clinical phase trials.

\begin{tabular}{cccc}
\hline Phases & Dosing & Number of subjects & Main goal of clinical phase \\
\hline Preclinical & Unrestricted & Not applicable & Testing in non-humans (efficacy, toxicities, pharmacokinetics) \\
0 & Subtherapeutic & About 10 & Pharmacokinetics and pharmacodynamics \\
IA/IB & Ascending doses & $20-100$ & Dose-ranging \\
IIA/IIB & Therapeutic dose & $100-300$ & Therapeutic effect \\
IIIA/IIIB & Therapeutic dose & $1000-2000$ & Long-term effects \\
IV & Therapeutic dose & Anyone seeking treatment & Research on data collected \\
V & No dosing & All reported use &
\end{tabular}


1993 and 2003, but major drug and biological product submissions to the FDA decreased. Investment required for one successful drug launch increased from \$1.1B in 1995-2000 to \$1.7B in 2000-2002. The critical path, which begins when candidate products are selected for development, was challenging, inefficient, and costly. Clinical failure included safety problems and lack of effectiveness. The concern was stagnation and declining innovation with a widening gap between knowledge and clinical use. A drug entering Phase I trials in 2000 was not more likely to come to market than one entering Phase I trials in 1985 [23]. Improvement in prediction of failure during early clinical trials saves in development costs and time to market [24]. The concept of exploratory investigation new drug (IND) studies was a result of this FDA analysis and can help with determining whether a defined mechanism of action can also be observed in humans, provide information on pharmacokinetics, select promising products from a group of candidates, and evaluate biodistribution. The purpose of these studies is to help in the go versus no-go decision making process of a drug's fate early in the development process using human models rather than relying on animal data.

Exploratory IND studies (also known as Phase 0 studies) are conducted early in clinical phase studies and involve limited human exposure and have no therapeutic or diagnostic intent. Doses are subtherapeutic and patients are monitored by the clinical researcher and involve about 10 study patients. Duration of a patient's participation is usually less than 1 week. Phamacodynamics and pharmacokinetics are studied. These trials are before the traditional dose escalation, safety, and tolerance studies, do not replace the Phase I clinical trials and do not indicate whether a therapy has a positive impact on the targeted pathology. These studies help in eliminating candidate therapies before they reach Phase I studies [25]-[27]. These trials were developed to shorten the critical path for drug development, to explore pharmacokinetic and pharmacodynamic profiles of IND's in humans, to help in accelerating identification of promising drugs, and to reduce development time and costs. Limitations of these trials include lack of therapeutic intent, motivation of patients to participate, may delay or exclude patients from other clinical trials that may have therapeutic intent, microdosing pharmacokinetics and relationship to therapeutic dose, and availability of sensitive analytical methods [28]. Attrition rates are high and only about $8 \%$ come to market.

\section{Phase I Clinical Trials}

A Phase I clinical trial evaluates the best way to administer a drug, its frequency and dose, the maximum tolerated dose (MTD), and side effects. Tolerability, pharmacokinetics, and pharmacodynamics are evaluated. These studies determine, most importantly, if the treatment is safe. Trials usually include 20 to 100 patients and are monitored by the clinical researcher. Doses are increased if there are no severe side effects and patients are tested to determine if he or she is responding to the therapy. These escalation dose studies are used to determine the best and safest dose that can be administered and is a fraction of the dose that caused harm during animal testing. Unnecessary exposure of subjects to subtherapeutic doses while maintaining safety and rapid accrual is the primary goal of Phase I trials [29]. Subjects, in most cases, are healthy volunteers although patients with a certain disease may be required. Contract research organizations usually conduct these studies and stipends may be given. Testing is usually sequential with data being reviewed after every patient or small group of patients.

Dose-toxicity and dose-efficacy curves are determined during this phase and include single ascending dose trials (Phase IA), multiple ascending dose trials (Phase IB), and food effect studies. Dose escalation methods may be rule-based or model-based. Rule-based designs do not stipulate any prior assumption of the dose-toxicity curve and allow escalation and de-escalation of the dose with diminishing fractions of the preceding dose depending on presence or absence of toxicity. They are easy to implement and do not require special software. The traditional $3+3$ design proceeds with cohorts of 3 patients. The starting dose is based on extrapolation from animal toxicological data. Increasing dose levels have been fixed in advance and usually follow a modified Fibonacci sequence in which the dosing increments become smaller as the dose increases [30]. If none of the patients experience a dose-limiting toxicity, 3 more patients will be treated at the next higher dose. If 1 of the patients experiences a dose-limiting toxicity, the same dose is repeated in 3 more patients. Dose escalation continues until at lease 2 patients from a cohort of 3 to 6 experience dose-limiting toxicities. Recommended dose for Phase II trials is defined as the dose level just below the toxic dose level.

Alternate rule-based dose escalation methods include the " $2+4$, ," " $3+3+3$," and " $3+1+1$ " ("best of five”) rules [31]. In the " $2+4$ " design, if one dose-limiting toxicity is observed in a first cohort of 2 patients, an additional cohort of 4 patients is added. The stopping rule is the same as for the " $3+3$ " studies. With the " $3+3+3$ " 
study, a third cohort of 3 patients is added if 2 of 6 patients in the first 2 cohorts have a dose-limiting toxicity. If at least 3 of 9 patients experience a dose-limiting toxicity, the study is terminated. The "best of 5" design requires that 1 additional patient is added if 1 or 2 dose-limiting toxicities are observed in the first 3 patients. Another patient is added if 2 dose-limiting toxicities are seen among the 4 treated patients. Escalation is continued if no dose-limiting toxicities are seen of 3,1 of 4 , or 2 of 5 patients. If 3 or more dose-limiting toxicities are seen, the trial is stopped.

Accelerated titration designs combine variations of the $3+3$ design and the model-based design. Patient assignment to doses is based on prespecified rules. Pharmacologically guided dose escalation is a variation of the $3+3$ design method. This assumes that animal model studies accurately reflect dose-limiting toxicities based on plasma drug concentrations. In the first stage, plasma exposure is extrapolated from preclinical data. Pharmacokinetic data are then obtained for each patient to determine subsequent dosing [32]. The isotonic regression model assumes toxicity is nondecreasing with dose and fits an isotonic regression to accumulated data. The dose given is that with estimated toxicity thought closest to the maximum tolerable toxicity [33]. The "rolling six design" allows for accrual of 2 to 6 patients concurrently onto a dose level based on the number of patients enrolled and evaluable, the number having dose-limiting toxicity (DLT), and the number still at risk of developing DLT [34]. This design is intended to shorten the study duration in which there is prior information about the dose range and is useful in pediatric populations. The "biased coin up-and-down design" requires that the treatment response or the toxicity evaluation is observed quickly, allocates a dose to each patient based on the toxicity information of the last completed subject and allows multiple patients to be concurrently studied [35]-[37]. Another rule-based design allows subsequent patients to be assigned to doses according to the toxicity outcomes at the current dose by calculating the toxicity probability interval under the beta-binomial model [38].

Model-based designs use statistical models that look for a dose level that produces a probability of doselimiting toxicity by using toxicity data from all enrolled patients to compute a more precise dose-toxicity curve. Bayesian models are commonly used. These models require an estimation of $\theta$ (characterizes the shape of the dose-toxicity curve.) Occurrence of toxicity results in adjustment of $\theta$ based on Bayes' theorem. These designs provide a confidence interval for recommended doses for Phase II clinical trials. The continual reassessment method was the first Bayesian model-based method used in Phase I clinical trial designs [39]. The initial estimate of $\theta$ is sought from experts familiar with the preclinical data or who have experience with similar drugs. Patients are treated at the dose thought to be closest to the MTD and estimation of the probability of a doselimiting toxicity is calculated for each new patient who enters the study until a prespecified condition is met at which time the trial is stopped. Modifications of this method have included treating the patient at the lowest started dose level, increasing the dose by only one prespecified level at a time, not allowing dose escalation for the immediate next patient if a previous patient experienced a dose-limiting toxicity, treating several patients at the same dose level and expanding the cohort of patients [40]-[43]. Escalation with overdose control was suggested as an alternative Bayesian approach to overcome the limitation of patients being exposed to high toxic doses [44]. Other model-based designs include time-to-event endpoint and the efficacy and toxicity methods. These model-based methods result in good estimations of the target probability of DLT at the recommended dose for Phase II clinical trials without treating too many patients at a suboptimal dose.

Dose-escalation strategies for trials of combinations of agents have included alternate escalation of the agents in the series of dose levels, simultaneous escalation of both agents, escalation of one agent to the recommended dose for Phase II trials while holding the other agent at a fixed dose, and escalation of one agent to the recommended dose for Phase II trials while holding the other agent at a low dose. In a review, Riviere and colleagues determined that $88 \%$ of the trials had a traditional or modified $3+3$ dose escalation design used. The calculated median DLT rate was 6\%. The authors recommended that the starting doses, dose levels, and dose-escalation steps must ensure patient safety, treat as few patients as possible at subtherapeutic doses, and identify optimal drug combinations for further evaluation [45]. No specific clinical trial designs have been formulated for molecularly targeted agents that have a proven relevant target and a validated method for measuring target inhibition.

\section{Phase II Clinical Trials}

Phase I/II dose finding studies determine the most successful dose (MSD) which is the dose which maximizes the product of the probability of seeing no toxicity together with the probability of seeing a therapeutic response. While a Phase I clinical study focuses on determining the MTD, Phase II studies evaluate potential efficacy and 
characterizes treatment benefit for the disease in a convincing manner. The intervention is not presumed to have any therapeutic effect whatsoever. These studies are performed on larger groups (100 to 300 subjects) and are designed to assess how well the drug works and to continue safety assessments. Therapeutic doses which were determined during Phase I are administered and patients are monitored by the clinical researcher. Trials are often conducted in a multi-institution setting. Phase II may be divided into Phase IIA which are pilot clinical trials to evaluate efficacy and safety in selected populations with the disease or condition to be treated, diagnosed or prevented (objectives may be on dose-response, type of patient, frequency of dosing, or other identifiers of safety and efficacy) and Phase IIB which are the most rigorous trials designed to demonstrate efficacy. The development process usually fails during this Phase II when the drug is discovered not to work as planned or to have toxic effects.

The Phase II design depends on the quality and adequacy of Phase I studies. A vulnerable aspect of both phases is the type of patient enrolled. Patients in Phase II trials generally have more exclusion criteria than those in Phase III trials. Case series and randomized clinical trial designs have been used. Single stage and multi-stage Phase II clinical trial designs are often developed on the basis that one endpoint is of interest. A commonly used Phase II design is based on the work of Gehan, a version of a two-stage design [46]. Other designs have more stages or a sequential aspect. Hybrid designs have been used to improve efficiency. In an update, Gehan reviewed statistical aspects of plans for Phase II cancer clinical trials including a minimum number of patients plan, a two-stage decision theory approach, a limited patient accrual plan, a predictive probability plan, and a one-sample multiple testing procedure plan. The author makes recommendations regarding the plan that best fits the needs of the study [47].

Adaptive clinical trial designs based on accumulated data at interim have also been used in Phase II clinical trials because of flexibility and efficiency. This design may allow the researcher to modify or redesign the trial while the study is ongoing. However, researchers have hesitated in their use-there is confusion with respect to definition, controversy regarding sample size re-estimation methods, and logistical barriers in using adaptive designs within existing trial frameworks [48]. In 2010, The FDA classified adaptive designs into "well understood" and "less well understood" categories [49]. "Well understood" designs have been in use for years with corresponding statistical methods that have been well established and the FDA is familiar with the study designs through the review of submissions using them. In the "less well understood" designs, relative merits and limitations have not been completely evaluated, valid statistical methods have not been developed, and the FDA does not have a lot of experience with submissions using the study designs. Chow et al. give a broader definition of adaptive design-one that allows adaptations in trial procedures and/or statistical procedures after initiation of the trial without undermining the validity and integrity of the trial [50]. Adaptive clinical trial designs include an adaptive randomization design, an adaptive group sequential design, a flexible sample size re-estimation design, a drop-the-losers design, an adaptive dose-finding design, a biomarker-adaptive design, an adaptive treatmentswitching design, an adaptive-hypothesis design, a Phase I/II or II/III adaptive seamless trial design and a multiple adaptive design.

\section{Phase III Clinical Trials}

Phase III trials are the full scale evaluation of treatment and are designed to compare efficacy of the new treatment with the standard treatment. These are the most rigorous and extensive type of scientific clinical investigation of a new treatment. This is the "pre-marketing phase" of clinical trials. These are usually the most expensive and time-consuming of the trials. The trials may be difficult to design and run. Large groups (100 to 3000 subjects) are recruited and trial designs have included randomized controlled trials (parallel design), uncontrolled trials (single treatment), historical controls, no-randomized concurrent trials, factorial designs, and group sequential designs. Patients are monitored by the clinical researcher and personal physician. Phase III clinical trials may be divided into Phase IIIA which are trials done after efficacy of the therapy is demonstrated but before regulatory submission of a New Drug Application (NDA) or other dossier and Phase IIIB which are conducted after submission of an NDA or other dossier but before approval and launch.

During the 1980's, the FDA published guidance documents that efficacy should be demonstrated by prolongation of life, improved health-related quality of life, or an established surrogate for one of these. If the new therapy results in a statistically significant improvement, the new treatment is usually approved for clinical use [51]. Traditional endpoints for trials have included overall survival, time to tumor progression, overall response rate, 
time to treatment failure and patient-reported outcomes. Overall survival has been the gold standard for the demonstration of clinical benefits. Subpart $\mathrm{H}$ allows for accelerated approval of drugs for serious and lifethreatening diseases where the drug demonstrates an advantage over available therapy. This is based on a surrogate endpoint that likely predicts clinical benefit. While randomized Phase III clinical trials have been the gold standard evidence for the approval of new drugs, problems associated with drug development have included limited clinical benefit in large RCT's, prediction of a successful Phase III trial from Phase II data, determination of toxicity, design of studies with drug combinations, and cost of the trial.

Ocana and colleagues suggest that adaptive designs in selected prescreened populations could reduce the limitations [52]. Statistical methods for the design and analysis of adaptive designs began in the 1990's [53] [54]. However, many of the designs are not standard and relate only to the application being considered. The experience of sponsors and regulators in planning, conducting and interpreting results using these designs is limited and interaction with regulating authorities early is crucial. In Europe, the European Medicines Agency offers developers of drugs and therapeutic devices scientific advice and protocol assistance [55]. In 2010, the FDA published guidance on adaptive design clinical trials [49]. Evaluators of adaptive clinical trial studies answer the following 6 questions: 1) Is there a good rationale and have alternative designs been considered? 2) Does the proposal fit well in the context of the development program and the data that will be available for the marketing authorization application? 3) Can the proposal be implemented without important damage to trial integrity? 4) Is the type I error rate controlled? 5) Has the potential bias of treatment effect estimates been evaluated? 6) Is the proposal practical and feasible [49]? These questions are also asked of other designs. The European Organisation for Research and Treatment of Cancer recognize that these designs can be advantageous, but warn that they must prevent bias that could be uncontrollable. Recommended techniques include randomisation, blinding, prospectively planned adaptations and upfront implementation of the process and firewalls needed to ensure restricted access to interim analysis results and blinding of staff involved in day-to-day trial proceedings [56].

\section{Phase IV Clinical Trials}

Upon authorization by the FDA, therapies determined to have proven safety, efficacy and quality may be made available to the general population. Patients and their physicians have expectations of benefit. However, not all safety or efficacy issues have been determined. The FDA requires continued evaluation after release to evaluate safety signs that may affect the benefit-risk ratio [57] [58]. These Phase IV studies include "all studies (other than routine surveillance) performed after drug approval and related to the approved indication" [55]. These are post-marketing surveillance studies. The focus of the trials is on how drugs work in the real world. Anyone seeking treatment from their physician may be treated with the therapy. Their personal physician monitors the results of treatment. Efficacy and detection of rare or long-term adverse effects over a much larger patient population and longer time period are evaluated, healthcare costs and outcomes are determined, and pharmacogenetics are studied. New clinical indications for a drug may be established and large number of patients and physicians are involved [59]. The FDA may require that a developer conduct a Phase IV trial as a stipulation for drug approval. Less than half of studies are completed or even initiated by developers [60]. Phase IV trials may result in a drug being removed from the market or restricted to certain indications.

Initially, these trials were run much like Phase III studies and were conducted for marketing purposes. Studies were done at institutions with investigators familiar with clinical trials and had inclusion and exclusion criteria similar to those of Phase III studies. Results did not reflect what would happen under normal conditions. As a result, innovative studies were designed to involve ordinary physicians in naive research communities. Goals have been broadened and include evaluation of specific pharmacological effects, establishing the incidence of adverse reactions, determining effects of long-term administration of a therapy, establishing a new clinical indication for the therapy, evaluation of the therapy in higher risk populations, etc. A main issue of concern is the mix of medical research and clinical practice [61].

Reported serious adverse drug reactions submitted to the FDA's Med Watch program have increased from 150,000 in 2000 to 370,000 in 2009 [62]. Physician and consumers or drug manufacturers submit these reports. Criticisms have included reliance on voluntary reporting of adverse events, trust in drug manufacturers to collect/evaluate/report drug safety data that may risk financial interests, and dependence on one government body to approve a drug and then require studies that might lead to withdrawal from the market [60] [63]. Proffered solutions have included large-scale simple RCTs with few eligibility and treatment criteria [64], preplanned me- 
taanalyses of a series of related trials [65], and establishment of a national health data network to evaluate post-marketing surveillance independent of the FDA-approval process [66].

\section{Phase V Clinical Trials}

This translational research is designed to "move from bench to bedside". Phase V clinical trials refer to comparative effectiveness research and community-based research. Research is done on data collected. All reported uses are evaluated. Patients are not monitored. Its main focus is to determine integration of a new therapy into wide spread clinical practice. Filed under: cornell cooperative extension, evidence-based living, policy, the learning center tagged with: cooperative extension programs, evaluation, evidence-based programs, research methods, research translation.

\section{Summary}

The final outcome of clinical trials is improved clinical medicine. Understanding the steps to bring a new therapy to the general population provides clinicians insight into their staged development and timeline to availability. By improving developmental strategies and studies, time to availability to the general public with resulting benefit should result in better patient outcomes and fewer morbidities.

\section{References}

[1] Browne, L.H. and Graham, P.H. (2014) Good Intentions and ICU-GCP: Trial Conduct Training Needs to Go beyond the ICH-GCP Document and include the Intention-to-Treat Principle. Clinical Trials, 11, 629-634. http://dx.doi.org/10.1177/1740774514542620

[2] Ohmann, C., Kuchinke, W., Canham, S., Lauritsen, J., Salas, N., Schade-Brittinger, C., et al. (2011) Standard Requirements for GCP-Compliant Data Management in Multinational Clinical Trials. Trials, 12, 85. http://dx.doi.org/10.1186/1745-6215-12-85

[3] Rock, E.P., Molloy, V.J. and Humphrey, J.S. (2010) GCP Data Quality for Early Clinical Development. Clinical Cancer Research, 16, 1756-1763. http://dx.doi.org/10.1158/1078-0432.CCR-09-3267

[4] Switula, D. (2000) Principles of Good Clinical Practice (GCP) in Clinical Research. Science and Engineering Ethics, 6, 71-77. http://dx.doi.org/10.1007/s11948-000-0025-z

[5] Vijayananthan, A. and Nawawi, O. (2008) The Importance of Good Clinical Practice Guidelines and Its Role in Clinical Trials. Biomedical Imaging and Intervention Journal, 4, e5. http://dx.doi.org/10.2349/biij.4.1.e5

[6] Chalmers, T.C., Smith, H., Blackburn, B., Silverman, B., Schroeder, B., Reitman, D., et al. (1981) A Method for Assessing the Quality of a Randomized Control Trial. Controlled Clinical Trials, 2, 31-49. http://dx.doi.org/10.1016/0197-2456(81)90056-8

[7] Haahr, M.T. and Hróbjartsson, A. (2006) Who Is Blinded in Randomized Clinical Trials? A Study of 200 Trials and a Survey of Authors. Clinical Trials, 3, 360-365.

[8] Jadad, A.R., Moore, R.A., Carroll, D., Jenkinson, C., Reynolds, J.M., Gavaghan, D.J., et al. (1996) Assessing the Quality of Reports of Randomizing Clinical Trials: Is Blinding Necessary? Controlled Clinical Trials, 17, 1-12. http://dx.doi.org/10.1016/0197-2456(95)00134-4

[9] Moher, D., Jadad, A.R., Nichol, G., Penman, M., Tugwell, P. and Walsh, S. (1995) Assessing the Quality of Randomized Controlled Trials: An Annotated Bibliography of Scales and Checklists. Controlled Clinical Trials, 16, 62-73. http://dx.doi.org/10.1016/0197-2456(94)00031-W

[10] Torgerson, D.J. and Roland, M. (1998) What Is Zelen’s Design. British Medical Journal, 316, 606-608. http://dx.doi.org/10.1136/bmj.316.7131.606

[11] E-Source Behavioral \& Social Sciences Research. Clinical Trials. www.esourceresearch.org

[12] Stanley, K. (2007) Design of Randomized Controlled Trials. Circulation, 115, 1164-1169. http://dx.doi.org/10.1161/CIRCULATIONAHA.105.594945

[13] Avins, A.L. (1998) Can Unequal Be More Fail? Ethics, Subject Allocation, and Randomised Clinical Trials. Journal of Medical Ethics, 24, 401-408. http://dx.doi.org/10.1136/jme.24.6.401

[14] Lachin, J.M., Matts, J.P. and Wei, L.J. (1988) Randomization in Clinical Trials: Conclusions and Recommendations. Controlled Clinical Trials, 9, 365-374. http://dx.doi.org/10.1016/0197-2456(88)90049-9

[15] Schulz, K.F. and Grimes, D.A. (2002) Generation of Allocation Sequences in Randomised Trials: Chances, Not Choice. The Lancet, 359, 515-519. http://dx.doi.org/10.1016/S0140-6736(02)07683-3 
[16] Sibbald, B. and Roland, M. (1998) Understanding Controlled Trials: Why Are Randomised Controlled Trials Important? BMJ, 316, 201-203. http://dx.doi.org/10.1136/bmj.316.7126.201

[17] Thall, P.F. and Wathen, J.K. (2007) Practical Bayesian Adaptive Randomization in Clinical Trials. European Journal of Cancer, 43, 859-866. http://dx.doi.org/10.1016/j.ejca.2007.01.006

[18] DeMets, D., Friedman, L. and Furberg, C. (2010) Fundamentals of Clinical Trials. 4th Edition, Springer, Berlin.

[19] Rogers, M.A. (2009) What Are the Phases of Intervention Research? American Speech-Language-Hearing Association, Rockville.

[20] Becich, M.J. (2007) Lessons Learned from the Shared Pathology Informatics Network (SPIN): A Scalable Network for Tanslational Research and Public Health. Journal of the American Medical Informatics Association, 14, 534-535. http://dx.doi.org/10.1197/jamia.M2477

[21] US Department of Health and Human Services Food and Drug Administration (2012) Innovation or Stagnation: Challenges and Opportunity on the Critical Path to New Medical Products.

http://www.fda.gov/oc/initiatives/criticalpath/whitepaper.html.

[22] Lloyd, I. (2002-2003) New Technologies, Products in Development, and Attrition Rates: R\&D Revolution Still around the Corner. PARAXEL’s Pharmaceutical R\&D Statistical Sourcebook. PAREXEL International Corporation, Waltham.

[23] Boston Consulting Group (2002-2003) A Revolution in R\&D: How Genomics and Genetics Will Affect Drug Development Costs and Times. PARAXEL's Pharmaceutical R\&D Statistical Sourcebook. PAREXEL International Corporation, Waltham.

[24] Kinders, R., Parchment, R.E., Ji, J., Kummar, S., Murgo, A.J., Gutierrez, M., et al. (2007) Phase 0 Clinical Trials in Cancer Drug Development: From FDA Guidance to Clinical Practice. Molecular Interventions, 7, 325-334. http://dx.doi.org/10.1124/mi.7.6.9

[25] Kummar, S., Kinders, R., Rubinstein, L., Parchment, R.E., Murgo, A.J., Collins, J., et al. (2007) Compressing Drug Development Timelines in Oncology Using Phase “0” Trials. Nature Reviews Cancer, 7, 131-139. http://dx.doi.org/10.1038/nrc2066

[26] Marchetti, S. and Schellens, J.H.M. (2007) The Impact of FDA and EMEA Guidelines on Drug Development in Relation to Phase 0 Trials. British Journal of Cancer, 97, 577-581. http://dx.doi.org/10.1038/sj.bjc.6603925

[27] Schellens, J.H.M. (2009) Phase 0 (Zero) Clinical Trials: More than Zero Benefits? European Journal of Cancer, 45, 728-729. http://dx.doi.org/10.1016/j.ejca.2009.01.022

[28] Le Tourneau, C., Lee, J.J. and Siu, L.L. (2009) Dose Escalation Methods in Phase I Cancer Clinical Trials. Journal of the National Cancer Institute, 101, 708-720. http://dx.doi.org/10.1093/jnci/djp079

[29] Storer, B.E. (1989) Design and Analysis of Phase I Clinical Trials. Biometrics, 45, 795-798. http://dx.doi.org/10.2307/2531693

[30] Rogatko, A., Schoeneck, D., Jonas, W., Tighiouart, M., Khuri, F.R. and Porter, A. (2007) Translation of Innovative Designs into Phase I Trials. Journal of Clinical Oncology, 25, 4982-4986. http://dx.doi.org/10.1200/JCO.2007.12.1012

[31] Storer, B.E. (2001) An Evaluation of Phase I Clinical Trial Designs in the Continuous Dose-Response Setting. Statistics in Medicine, 20, 2399-2408. http://dx.doi.org/10.1002/sim.903

[32] Graham, M.A. and Workman, P. (1992) The Impact of Pharmacokinetically Guided Dose Escalation Strategies in Phase I Clinical Trials: Critical Evaluation and Recommendations for Future Studies. Annals of Oncology, 3, 339-347.

[33] Leung, D.H. and Wang, Y. (2001) Isotonic Designs for Phase I Trials. Controlled Clinical Trials, 22, 126-138. http://dx.doi.org/10.1016/S0197-2456(00)00132-X

[34] Skolnick, J.M., Barrett, J.S., Jayaraman, B., Patel, D. and Adamson, P.C. (2008) Shortening the Timeline of Pediatric Phase I Trials: The Rolling Six Design. Journal of Clinical Oncology, 26, 190-195. http://dx.doi.org/10.1200/JCO.2007.12.7712

[35] Durham, S.D., Flournoy, N. and Rosenberger, W.F. (1997) A Random Walk Rule for Phase I Clinical Trials. Biometrics, 53, 745-760. http://dx.doi.org/10.2307/2533975

[36] Ivanova, A., Montazer-Haghighi, A., Mohanty, S.G. and Durham, S.D. (2003) Improved Up-and-Down Designs for Phase I Trials. Statistics in Medicine, 22, 69-82. http://dx.doi.org/10.1002/sim.1336

[37] Stylianou, M. and Follmann, D.A. (2004) The Accelerated Biased Coin Up-and-Down Design in Phase I Trials. Journal of Biopharmaceutical Statistics, 14, 249-260. http://dx.doi.org/10.1081/BIP-120028518

[38] Ji, Y., Li, Y. and Nebiyou Bekele, B. (2007) Dose-Finding in Phase I Clinical Trials Based on Toxicity Probability Intervals. Clinical Trials, 4, 235-244. http://dx.doi.org/10.1177/1740774507079442

[39] O’Quigley, J., Pepe, M. and Fisher, L. (1990) Continual Reassessment Method: A Practical Design for Phase I Clinical 
Trials in Cancer. Biometrics, 46, 33-48. http://dx.doi.org/10.2307/2531628

[40] Faries, D. (1994) Practical Modifications of the Continual Reassessment Method for Phase I Cancer Clinical Trials. Journal of Biopharmaceutical Statistics, 4, 147-164. http://dx.doi.org/10.1080/10543409408835079

[41] Goodman, S.N., Zahurk, M.L. and Piantadosi, S. (1995) Some Practical Improvements in the Continual Reassessment Method for Phase I Studies. Statistics in Medicine, 14, 1149-1161. http://dx.doi.org/10.1002/sim.4780141102

[42] Moller, S. (1995) An Extension of the Continual Reassessment Methods Using a Preliminary Up-and-Down Design in a Dose Finding Study in Cancer Patients in Order to Investigate a Greater Range of Doses. Statistics in Medicine, 14, 911-922. http://dx.doi.org/10.1002/sim.4780140909

[43] Piantadosi, S., Fisher, J.D. and Grossman, S. (1998) Practical Implementation of a Modified Continual Reassessment Method for Dose-Finding Trials. Cancer Chemotherapy and Pharmacology, 41, 429-436. http://dx.doi.org/10.1007/s002800050763

[44] Rogatko, A., Babb, J.S., Tighiouart, M., Khuri, F.R. and Hudes, G. (2005) New Paradigm in Dose-Finding Trials: Patient-Specific Dosing and Beyond Phase I. Clinical Cancer Research, 11, 5342-5346. http://dx.doi.org/10.1158/1078-0432.CCR-05-0458

[45] Riviere, M.K., Le Tourneau, C., Paoletti, X., Dubois, F. and Zohar, S. (2014) Designs of Drug-Combination Phase I Trials in Oncology: A Systematic Review of the Literature. Annals of Oncology. http://dx.doi.org/10.1093/annonc/mdu516

[46] Gehan, E.A. (1961) The Determination of the Number of Patients Required in a Preliminary and a Follow-Up Trial of a New Chemotherapeutic Agent. Journal of Chronic Diseases, 13, 346-353. http://dx.doi.org/10.1016/0021-9681(61)90060-1

[47] Gehan, E.A. (1986) Update on Planning of Phase II Clinical Trials. Drugs under Experimental and Clinical Research, 12, 43-50.

[48] Coffey, C.S. and Kairalla, J.A. (2008) Adaptive Clinical Trials: Progress and Challenges. Drugs in R\&D, 9, $229-242$. http://dx.doi.org/10.2165/00126839-200809040-00003

[49] US Food and Drug Administration (2010) Draft Guidance for Industry-Adaptive Design Clinical Trials for Drugs and Biologics. US Food Drug Admin, Rockville.

[50] Chow, S.C., Chang, M. and Pong, A. (2005) Statistical Consideration of Adaptive Methods in Clinical Development. Journal of Biopharmaceutical Statistics, 15, 575-591. http://dx.doi.org/10.1081/BIP-200062277

[51] Pazdur, R. (2008) Endpoints for Assessing Drug Activity in Clinical Trials. The Oncologist, 13, 19-21. http://dx.doi.org/10.1634/theoncologist.13-S2-19

[52] Ocana, A., Amir, E., Vera-Badillo, F., Seruga, B. and Tannock, I.F. (2013) Phase III Trials of Targeted Anticancer Therapies: Redesigning the Concept. Cancer Research, 19, 4931-4940.

[53] Bauer, P. and Kohne, K. (1994) Evaluation of Experiments with Adaptive Interim Analyses. Biometrics, 50, $1029-1041$. http://dx.doi.org/10.2307/2533441

[54] Proschan, M.A. and Hunsberger, S.A. (1995) Designed Extension of Studies Based on Conditional Power. Biometrics, 51, 1315-1324. http://dx.doi.org/10.2307/2533262

[55] Elsäßer, A., Regnstrom, J., Vetter, T., Koenig, F., Hemmings, R.J., Greco, M., et al. (2014) Adaptive Clinical Trial Designs for European Marketing Authorization: A Survey of Scientific Advice Letters from the European Medicines Agency. Trials, 15, 383. http://dx.doi.org/10.1186/1745-6215-15-383

[56] Mauer, M., Collette, L. and Bogaerts, J., European Organisation for Research and Treatment of Cancer (EORTC) Statistics Department (2012) Adaptive Designs at European Organisation for Research and Treatment of Cancer (EORTC) with a Focus on Adaptive Sample Size Re-Estimation Based on Interim-Effect Size. European Journal of Cancer, 48, 1386-1391. http://dx.doi.org/10.1016/j.ejca.2011.12.024

[57] Committee on Ethical and Scientific Issues in Studying the Safety of Approved Drugs (2012) Ethical and Scientific Issues in Studying the Safety of Approved Drugs. Institute of Medicine of the National Academics, Washington DC.

[58] Mello, M.M., Goodman, S.N. and Faden, R.R. (2012) Ethical Considerations in Studying Drug Safety-The Institute of Medicine Report. The New England Journal of Medicine, 367, 959-964. http://dx.doi.org/10.1056/NEJMhle1207160

[59] Bernabe, R.D.L.C., van Thiel, G.J.M.W., Raaijmakers, J.A.M. and van Delden, J.J.M. (2014) The Fiduciary Obligation of the Physician-Researcher in Phase IV Trials. BMC Medical Ethics, 15, 11. http://dx.doi.org/10.1186/1472-6939-15-11

[60] Fontanarosa, P.B., Rennie, D. and DeAngelis, C.D. (2004) Postmarketing Surveillance-Lack of Vigilance, Lack of Trust. Journal of the American Medical Association, 292, 2647-2650. http://dx.doi.org/10.1001/jama.292.21.2647

[61] van Thiel, G.J.M.W. and van Delden, J.J.M. (2008) Phase IV Research: Innovation in Need of Ethics. Journal of Medical Ethics, 34, 415-416. http://dx.doi.org/10.1136/jme.2007.023911 
[62] Administration USFDA (2011) AERS Patient Outcomes by Year 2010.

http://www.fda.gov/Drugs/GuidanceComplianceRegulatoryInformation/Surveillance/AdverseDrugEffects/ucm070461. $\underline{\mathrm{htm}}$

[63] Strom, B.L. (2004) Potential for Conflict of Interest in the Evaluation of Suspected Adverse Drug Reactions: A Counterpoint. Journal of the American Medical Association, 292, 2643-2646. http://dx.doi.org/10.1001/jama.292.21.2643

[64] Hennekens, C.H. and Demets, D. (2009) The Need for Large-Scale Randomized Evidence without Undue Emphasis on Small Trials, Meta-Analyses, or Subgroup Analyses. Journal of the American Medical Association, 302, 2361-2362. http://dx.doi.org/10.1001/jama.2009.1756

[65] Berlin, J.A. and Colditz, G.A. (1999) The Role of Meta-Analysis in the Regulatory Process for Foods, Drugs, and Devices. Journal of the American Medical Association, 281, 830-834. http://dx.doi.org/10.1001/jama.281.9.830

[66] Maro, J.C., Platt, R., Holmes, J.H., Strom, B.L., Hennessy, S., Lazarus, R., et al. (2009) Design of a National Distributed Health Data Network. Annals of Internal Medicine, 151, 341-344.

http://dx.doi.org/10.7326/0003-4819-151-5-200909010-00139

\section{Abbreviations}

API: Active Pharmaceutical Ingredient;

DLT: Dose-Limiting Toxicity;

FDA: Food and Drug Administration;

GCP: Good Clinical Practice;

IND: Investigation New Drug;

MSD: Most Successful Dose;

MTD: Maximum Tolerated Dose;

NDA: New Drug Application;

NIH: National Institutes of Health;

NOAEL: No Observed Adverse Effect Level;

RCP: Randomized Clinical Trials;

US Food and Drug Administration: United States Food and Drug Administration;

US Pharmaceutical R \& D: United States Pharmaceutical Research and Development. 
Scientific Research Publishing (SCIRP) is one of the largest Open Access journal publishers. It is currently publishing more than 200 open access, online, peer-reviewed journals covering a wide range of academic disciplines. SCIRP serves the worldwide academic communities and contributes to the progress and application of science with its publication.

Other selected journals from SCIRP are listed as below. Submit your manuscript to us via either submit@scirp.org or Online Submission Portal.
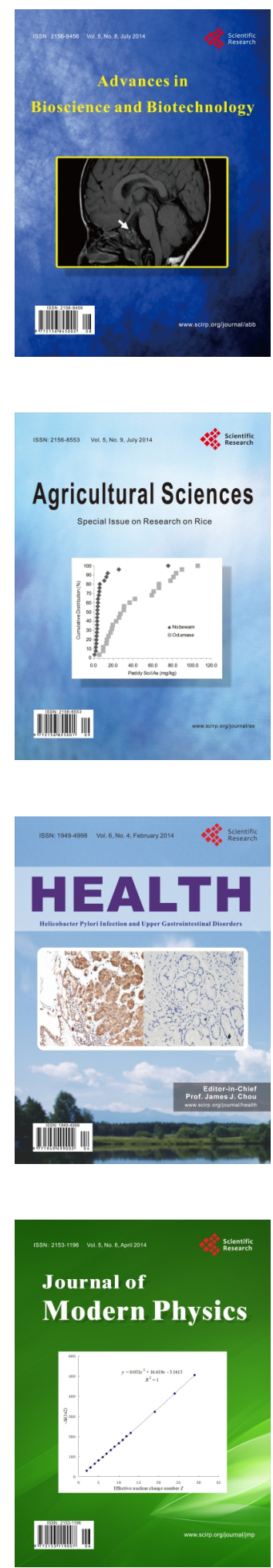
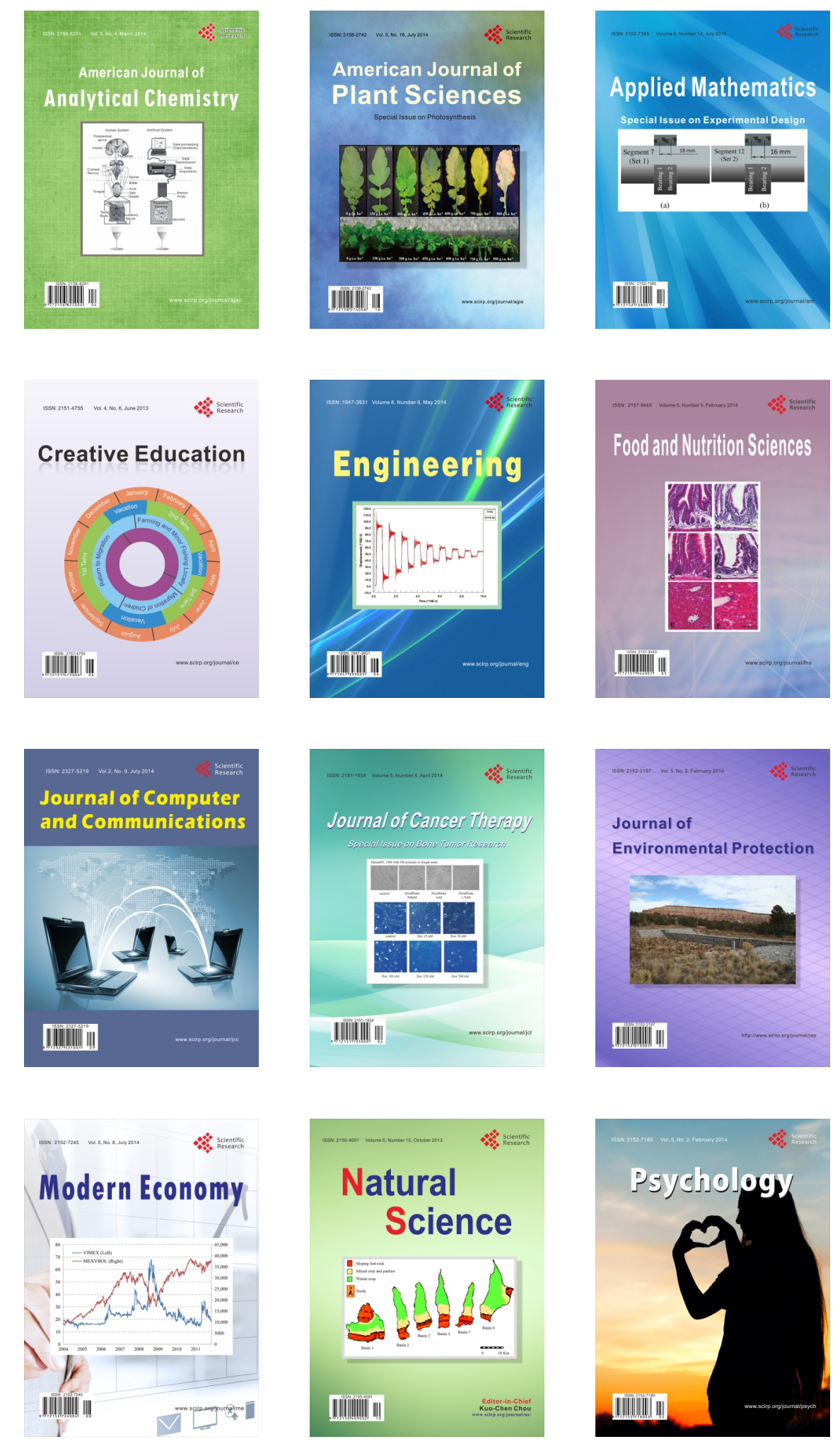